\title{
ARTICLE
}

Received 9 May 2014 | Accepted 23 Sep 2014 | Published 30 Oct $2014 \quad$ DOI: 10.1038/ncomms6349

\section{Signatures of Dirac fermion-mediated magnetic order}

\author{
Paolo Sessi', Felix Reis ${ }^{1}$, Thomas Bathon', Konstantin A. Kokh²,3, Oleg E. Tereshchenko ${ }^{3,4}$ \& Matthias Bode ${ }^{1,5}$
}

The spin-momentum locking of topological states offers an ideal platform to explore novel magnetoelectric effects. These intimately depend on the ability to manipulate the spin texture in a controlled way. Here we combine scanning tunnelling microscopy with single-atom deposition to map the evolution of topological states under the influence of different magnetic perturbations. We obtain signatures of Dirac fermion-mediated magnetic order for extremely dilute adatom concentrations. This striking observation is found to critically depend on the single adatoms' magnetic anisotropy and the position of the Fermi level. Our findings open new perspectives in spin engineering topological states at the atomic scale and pave the way to explore novel spin-related topological phenomena with promising potential for applications.

\footnotetext{
${ }^{1}$ Physikalisches Institut, Experimentelle Physik II, Universität Würzburg, Am Hubland, D-97074 Würzburg, Germany. ${ }^{2}$ V.S. Sobolev Institute of Geology and Mineralogy, Siberian Branch, Russian Academy of Sciences, Novosibirsk 630090, Russia. ${ }^{3}$ Novosibirsk State University, Novosibirsk 630090, Russia.

${ }^{4}$ A.V. Rzanov Institute of Semiconductor Physics, Siberian Branch, Russian Academy of Sciences, Novosibirsk 630090, Russia. ${ }^{5}$ Wilhelm Conrad Röntgen-Center for Complex Material Systems (RCCM), Universität Würzburg, Am Hubland, D-97074 Würzburg, Germany. Correspondence and requests for materials should be addressed to P.S. (email: paolo.sessi@physik.uni-wuerzburg.de).
} 
$\mathrm{M}$ anipulating unconventional spin textures represents a frontier towards quantum spintronics. Prominent systems showing novel properties associated with the spin degree of freedom are magnetic skyrmions ${ }^{1}$ and the recently discovered topological insulators (TIs) ${ }^{2,3}$. TIs, in particular, offer an ideal platform to explore several exotic phenomena which, in addition to being relevant to both condensed matter and highenergy physics ${ }^{4-6}$, are expected to have direct applications in devices with unique functionalities ${ }^{7-9}$. The promise of this new class of materials is related to their unconventional electronic properties. Contrary to other 'trivial' two-dimensional (2D) systems hosting massless Dirac fermions such as graphene ${ }^{10}$, topological states are robust against small perturbations and cannot be destroyed by the presence of defects and adsorbates as long as time-reversal (TR) symmetry is preserved ${ }^{11,12}$. Owing to strong spin-orbit coupling, the spin is perpendicularly locked to the momentum, resulting in a chiral spin texture ${ }^{13}$ that forbids backscattering ${ }^{14,15}$. This spin-momentum locking gives rise to electric charging of the magnetic texture offering new opportunities to explore topological magnetoelectric effects impossible with other semiconductor materials. The fundamental requirement for their experimental realization is intimately related to the capability of manipulating the spin texture in a controlled way, as it can be achieved through the interaction of topological states with magnetic moments.

Although numerous studies aimed to shed light on the role played by magnetic perturbations, contradictory results have been obtained and a clear picture is still missing ${ }^{16-25}$. Results published so far seem to suggest that surface- and bulk-doped samples behave quite differently. Namely, an opening of the Dirac gap with the onset of magnetic order was exclusively reported for bulk-doped samples ${ }^{16,18}$, while the Dirac cone remained intact for most studies employing surface doping $22-25$.

This is in contradiction to the theory which predicts that, because of the absence of scattering into the bulk, surfaceconfined Dirac fermions can mediate magnetic interaction over distances of several nanometres via the Ruderman-KittelKasuya-Yosida (RKKY) interaction ${ }^{26,27}$. This means that topological states are expected to significantly alter their spin texture and eventually establish magnetic order for very dilute magnetic adatom concentrations. If experimentally verified, it would allow for atomic level control of the spin texture of topological states.

To date, the experimental study of the interaction of surface magnetic moments with topological states has predominantly been performed by angle-resolved photoemission spectroscopy (ARPES) $)^{21-23}$ and X-ray magnetic circular dichroism $^{24,25}$. Although providing invaluable contributions towards the understanding of the interaction of magnetic moments with topological states, these spatially averaging techniques do not give access to local phenomena. The broadening observed in the ARPES data, for example, may be indicative of an inhomogeneous surface magnetization. Indeed-even if long-range magnetic order is absent-shortlength- and temporal-scale spin-ordered states might still exist but cancel each other and may therefore remain undetected by spatially averaging techniques.

Here we combine low-temperature scanning tunnelling microscopy with single-adatom deposition technique to directly map the evolution of the electronic properties of topological states under the influence of different magnetic perturbations. We reveal signatures of surface magnetic order as soon as the magnetic moments couple ferromagnetically. By using different magnetic elements and coverages, we find that this striking observation crucially depends on two parameters: single adatoms magnetic anisotropy direction and energy-level alignment.

\section{Results}

Scattering scenario on TIs. To obtain detailed information on the role played by magnetic perturbations, we employed energyresolved Fourier-transformed (FT) quasi-particle interference (QPI) mapping. This technique makes use of the standing wave pattern generated by the coherent scattering of electronic states at surface defects, such as vacancies, adsorbates, impurities or step edges. First employed to detect coherent scattering of conventional surface states on noble metals ${ }^{28}$, it is a powerful technique to investigate the scattering properties of surfaces. It has been successfully applied to study high-temperature superconductors ${ }^{29}$ as well as heavy-fermion systems ${ }^{30,31}$. Application of QPI to surfaces with non-degenerate spin bands has been pioneered on the $\mathrm{Bi}(110)$ surface $^{32}$ and has recently been extended to study spin-dependent scattering processes in Rashba systems ${ }^{33}$ and TIs $^{14,15,19}$.

For elastic scattering, the relation $\mathbf{k}_{\mathrm{f}}=\mathbf{k}_{\mathrm{i}}+\mathbf{q}$ must be fulfilled, where $\mathbf{q}$ is the scattering vector and $\mathbf{k}_{\mathrm{i}}$ is the wave vector of the incident Bloch wave that scatters into $\mathbf{k}_{\mathrm{f}}$ on the same constantenergy cut. If the initial and final states are non-orthogonal, they interfere resulting in a standing wave characterized by a spatial periodicity equal to $2 \pi /|\mathbf{q}|$, which is imaged by energy-resolved differential conductivity $(\mathrm{d} I / \mathrm{d} U)$ maps. FT translates the realspace information into reciprocal space, thereby providing direct insight into scattering processes ${ }^{34}$.

The typical scattering scenario on TIs, with backscattering forbidden by TR symmetry, is schematically represented in Fig. 1a. The situation changes as soon as magnetic adatoms are present on the surface. In this case, electrons can flip their spin during a scattering event, thereby allowing backscattering. Since eigenstates of electrons with opposite spin directions are orthogonal, their initial and final state wave functions cannot interfere (Fig. 1b). As the concentration of magnetic moments exceeds a certain threshold, they can couple because of the $2 \mathrm{D}$ RKKY interaction mediated by the Dirac fermions. Consequently, topological states are expected to acquire a nonvanishing spin a

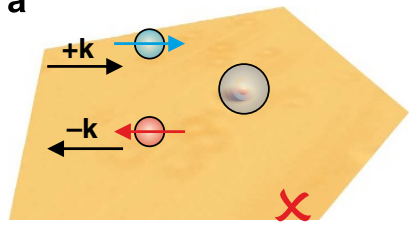

b

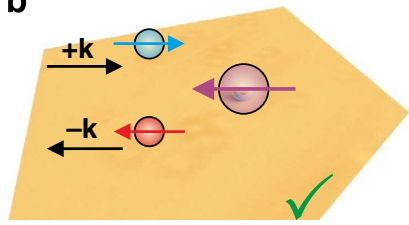

C

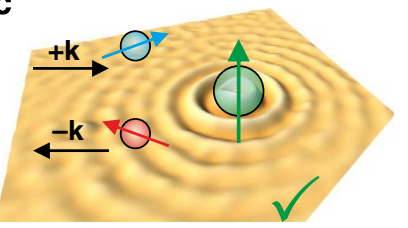

Figure 1 | Backscattering processes on topological insulator surfaces. While backscattering events are forbidden by TR symmetry on a pristine topological insulator surface, as illustrated in $\mathbf{a}$, the presence of magnetic impurities enables spin-flip processes that lead to backscattering (b). Since, however, orthogonal spin states cannot interfere, backscattering events contribute to the QPI signal only when TR symmetry is broken and magnetic moments become coupled through indirect exchange mediated by Dirac fermions, which acquire a nonvanishing spin component along the magnetization direction (c). Black arrows refer to the propagation directions, while coloured arrows indicate the spin directions. 
component along the magnetization direction, thereby allowing incoming and outgoing electrons to interfere (Fig. 1c). This corresponds to the moment when TR symmetry breaks, which in FT-QPI is signalled by the appearance of scattering vectors corresponding to backscattering events.

Transition metal atom adsorption and energy-level alignment. Figure $2 \mathrm{a}, \mathrm{b}$ shows two constant-current images taken on the very same sample region before and after Co deposition as spotlighted by arrows pointing at some characteristic defects. The same procedure has been applied for Mn (see Supplementary Fig. 1). Figure $2 \mathrm{c}-\mathrm{e}$ reports scanning tunnelling spectroscopy (STS) data obtained on the pristine, Co- and $\mathrm{Mn}$-doped $\mathrm{Bi}_{2} \mathrm{Te}_{3}$, respectively. For both Co and $\mathrm{Mn}$ the coverage corresponds to $\sim 1 \%$ of a monolayer (ML). Close inspection of constant-current images reveals two distinct topographical features appearing after the deposition as also observed for other $3 d$ metals $^{24}$. They correspond to single atoms adsorbed in hollow sites of the surface Te layer that are inequivalent with respect to the lattice of the subsurface Bi layer (see Supplementary Fig. 2).

The comparison of STS data taken before and after Co or Mn deposition reveals a rigid shift towards negative energies, which amounts to $\sim 100 \mathrm{meV}$ in either case, indicating a downwards band bending that results in an n-doped surface. The position of the valence band maximum and the conduction band minimum was determined following the procedure presented in ref. 35 (see Supplementary Fig. 3). STS spectra obtained by positioning the tip on top of the adatoms can be found in Supplementary Fig. 4. Note that for both adatom species, Co and Mn, the Fermi level is energetically located within the bulk gap at this adatom concentration. If there was a gap opening in the topological states, it would be invisible to STS being the Dirac point lower than the valence band maximum, as reported for ferromagnetic bulk-doped samples ${ }^{17,19}$.

Spectroscopic mapping of scattering channels-low coverages. Figure 3a-c reports energy-resolved QPI maps with the respective FT for pristine, Co- and Mn-doped $\mathrm{Bi}_{2} \mathrm{Te}_{3}$ obtained over the respective regions displayed in Fig. 2. QPI maps obtained on several pristine surfaces always showed the same behaviour, indicating the high homogeneity of our crystals. The pristine $\mathrm{Bi}_{2} \mathrm{Te}_{3}$ displayed in Fig. 3 refers to the very same sample region before Co evaporation. Data obtained before Mn deposition can be found in Supplementary Fig. 5 .

The FT-QPI maps exhibit sixfold symmetric peaks oriented along the $\Gamma-\mathrm{M}$ direction. They correspond to scattering vector $\mathbf{q}_{2}$ (see Fig. 3d), which connects parallel segments of next-nearest neighbour valleys of the warped equipotential surface and is routinely found on pristine ${ }^{15}$ and bulk-doped TIs ${ }^{19,36}$. Note that none of the FT QPI maps in Fig. 3 shows an intensity maximum in the $\Gamma-\mathrm{K}$ direction, indicating that backscattering is absent within the energy range detected here, that is, far above the Fermi level. Quantitative analysis of these data allows obtaining the energy dispersion relation of the Dirac cone along the $\Gamma-\mathrm{K}$ direction. Results are summarized in Fig. 3e. A linear fit provides the following values for the Dirac point: $E_{\mathrm{D}}=-155 \mathrm{meV}$, $E_{\mathrm{D}}=-245 \mathrm{meV}$ and $E_{\mathrm{D}}=-258 \mathrm{meV}$ for pristine, Co- and Mn-doped $\mathrm{Bi}_{2} \mathrm{Te}_{3}$, respectively.

This scattering scenario profoundly changes for states close to the Fermi level when Mn is present on the surface as reported in
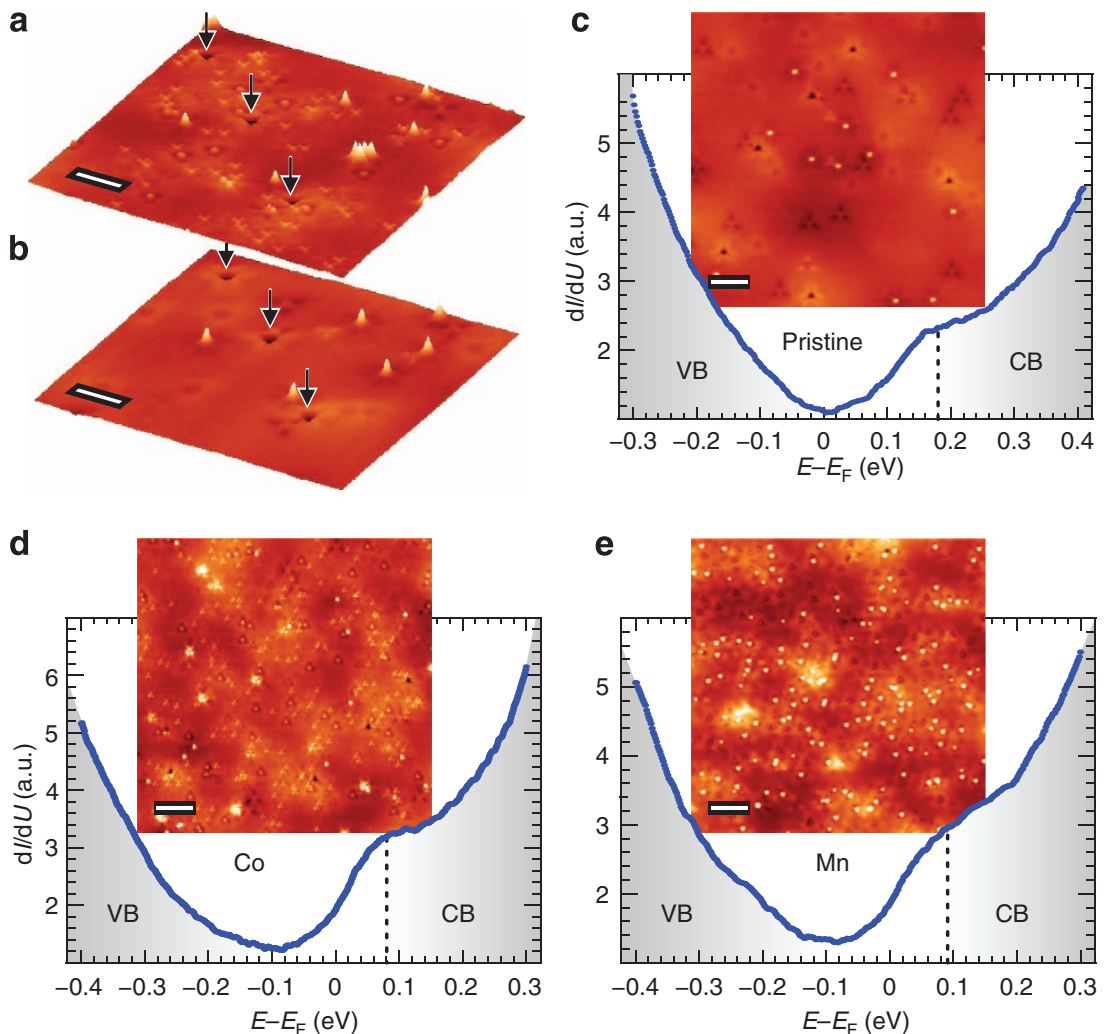

Figure 2 | Pristine and magnetic surface-doped $\mathbf{B i}_{\mathbf{2}} \mathbf{T e}_{\mathbf{3}}$. Constant current images taken on the very same sample region (a) before and (b) after deposition of $\mathrm{Co}$ atoms. Arrows point at surface defects used as markers. White scale bars correspond to $5 \mathrm{~nm}$. (c-e) Scanning tunneling spectroscopy curves taken on pristine, Co-doped and Mn-doped surfaces, respectively. Each inset reports the corresponding constant-current images over which scanning tunnelling spectra have been acquired at off-dopant locations. White scale bars correspond to $10 \mathrm{~nm}$. Scanning parameters of $I=50 \mathrm{pA}$ and $U=-0.3 \mathrm{~V}$ were used. 
a
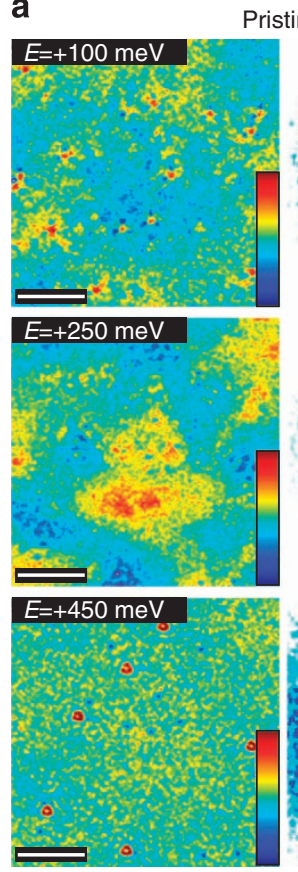

d

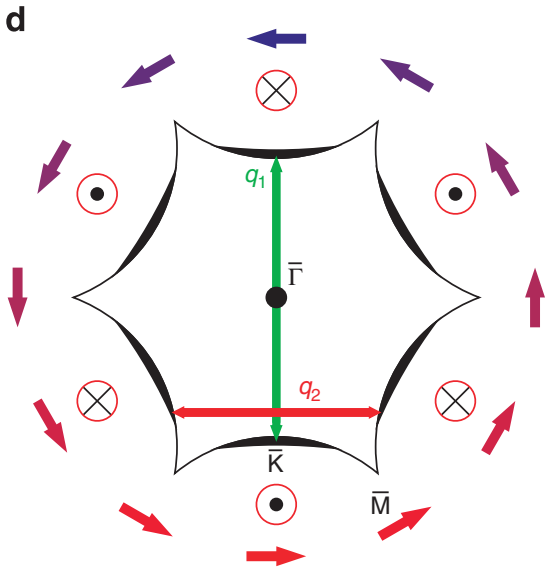

b
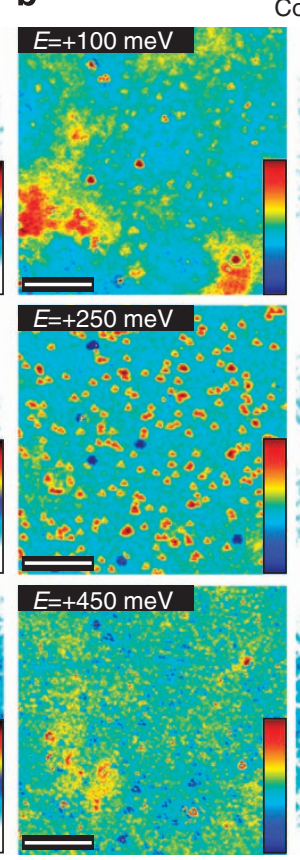

Co
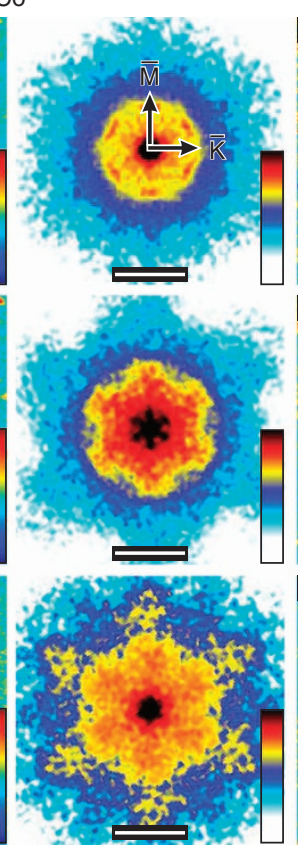

C
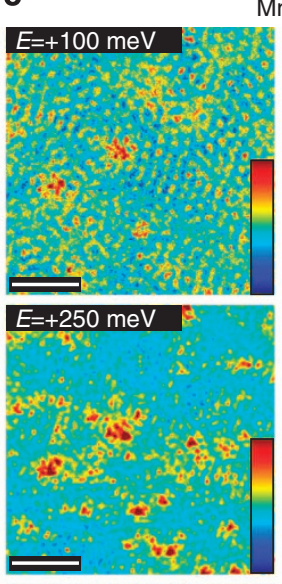

$E=+450 \mathrm{meV}$
$\mathrm{Mn}$

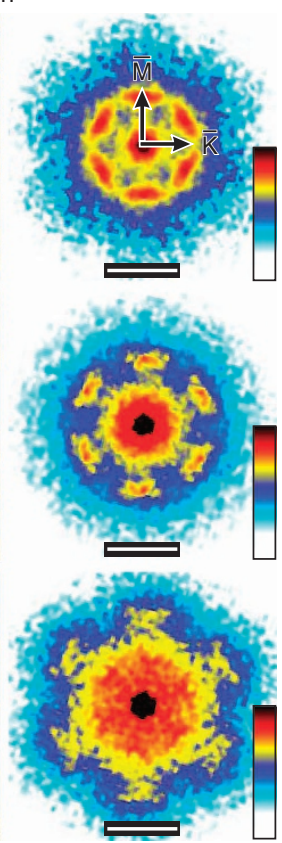

e

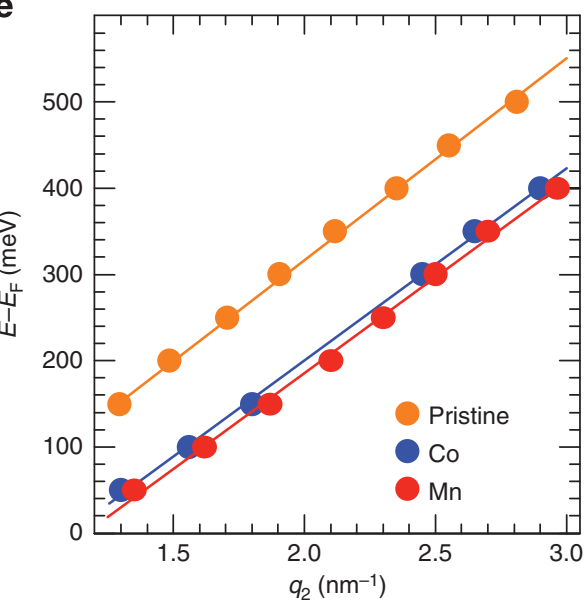

Figure 3 | Spectroscopic mapping of scattering channels. dl/dU maps with corresponding FT obtained at different energies on the (a) pristine, (b) Co- and (c) Mn-surface doped $\mathrm{Bi}_{2} \mathrm{Te}_{3}$. The labelled arrows in the FT indicate the directions of the surface Brillouin zone. The white scale bars correspond to $20 \mathrm{~nm}$ in the $\mathrm{dl} / \mathrm{d} U$ maps and $2.5 \mathrm{~nm}^{-1}$ in the FT panels. The intensity of the $\mathrm{d} l / \mathrm{d} U$ signal is represented by the colour scale with red (black) and blue (white) referring to high and low signals in $\mathrm{dl} / \mathrm{dU}$ maps (FTs), respectively. (d) Schematic illustration of the scattering scenario on $\mathrm{Tl}$ surfaces. The snowflake shape of the constant energy cut is the result of warping effects. Scattering vectors are indicated by $\mathbf{q}_{\mathbf{1}}$ and $\mathbf{q}_{\mathbf{2}}$. $\mathbf{q}_{\mathbf{1}}$ is forbidden in pristine $\mathrm{Bi}_{2} \mathrm{Te}_{3}$ by TR symmetry, while it becomes allowed once magnetic atoms are present on the surface. However, it can be observed by FT-QPI experiments only when the incoming and outgoing electrons have nonorthogonal spin states, that is, magnetic order is present on the surface. (e) Energy dispersion of the scattering vector $\mathbf{q}_{\mathbf{2}}$, the only one usually observed on TI surfaces. The plot was obtained by analysing the FT-QPI pattern at different energies. The straight lines are linear fits to the data.

Fig. 4. Note that because of the negative energy shift introduced by the surface dopants (see above), comparison of these FT-QPI maps with those obtained on the pristine surface should be carried out with respect to the panel corresponding to an energy of $+100 \mathrm{meV}$ in Fig. 2a. Any change in the scattering scenario and the consequent appearance of new scattering vectors can be thus unambiguously attributed to the effect of the magnetic impurities introduced on the surface. While no clear scattering channels can be identified once Co adatoms are deposited on the surface (see Fig. 4a), a new scattering vector pointing along $\Gamma-\mathrm{K}$ direction appears for Mn (see Fig. 4b), with an intensity that decreases by moving towards higher energies. Its origin can be understood by direct comparison with the ARPES constant- energy cut reported in Fig. $4 \mathrm{c}$ independently obtained on a $\mathrm{Bi}_{2} \mathrm{Te}_{3}$ sample with a Dirac point located at approximately $E_{\mathrm{D}}=-250$ $\mathrm{meV}$, as in the present case ${ }^{3}$. Its inspection shows that the direction and length of the new feature in our FT-QPI maps correspond to backscattering events $\mathbf{q}_{\mathbf{1}}$ (refs 16,19), forbidden by TR symmetry on pristine TIs.

Note that, although in QPI experiments the presence of anisotropic scattering centres may result in additional features appearing in the FT, we can exclude that the additional scattering vector is caused by the shape of the dopants. Indeed, the Mn atom's slightly triangular appearance would result in intensity along the $\Gamma-\mathrm{M}$ direction (see Supplementary Material in ref. 36), further suppressing the signal along $\Gamma-\mathrm{K}$. Furthermore, the 
a

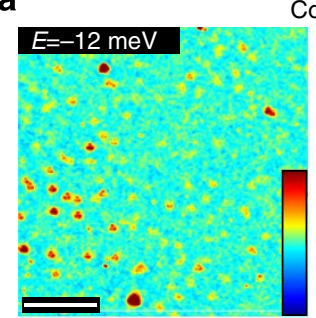

$E=+2 \mathrm{meV}$

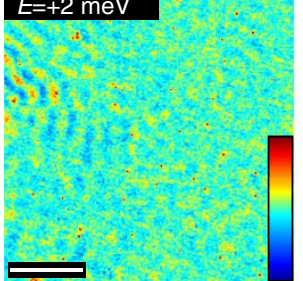

$E=+12 \mathrm{meV}$

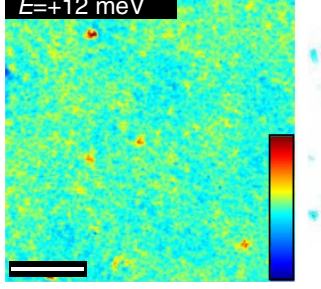

C

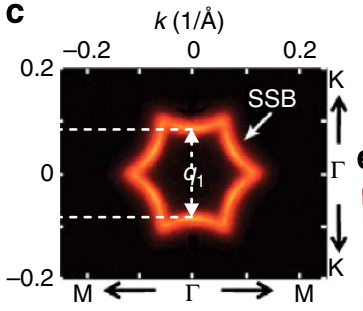

d

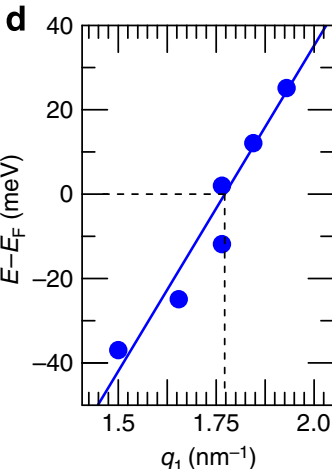

b

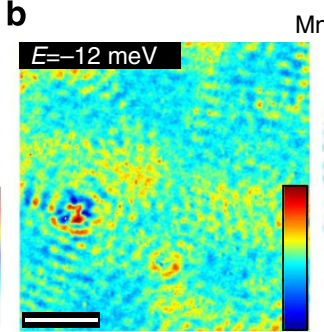

$\mathrm{Mn}$
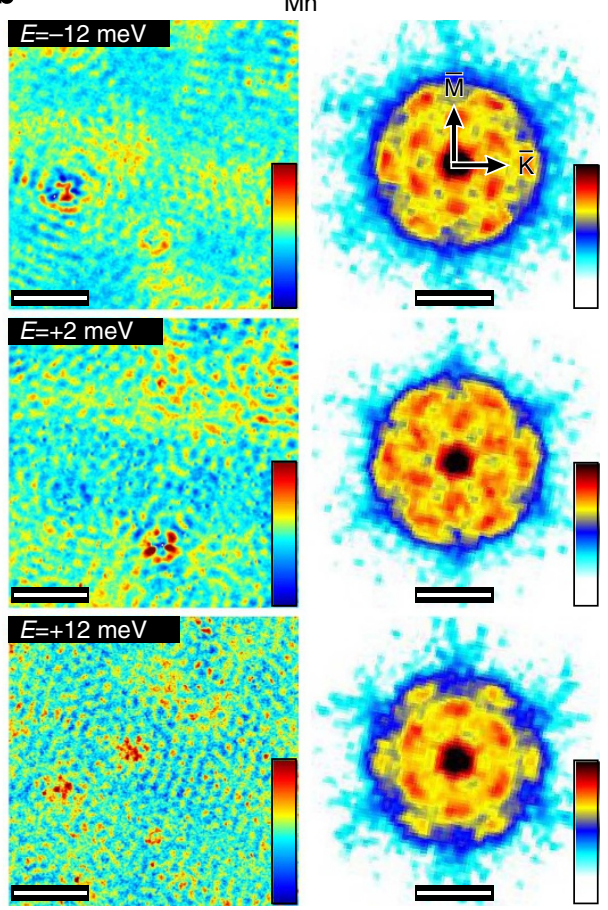
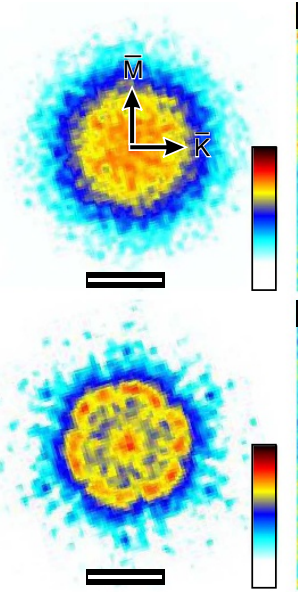

g

f

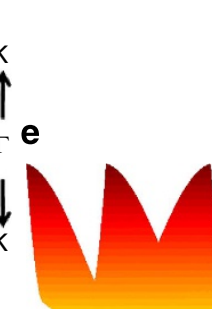

Magn. $x$

Magn. $z$
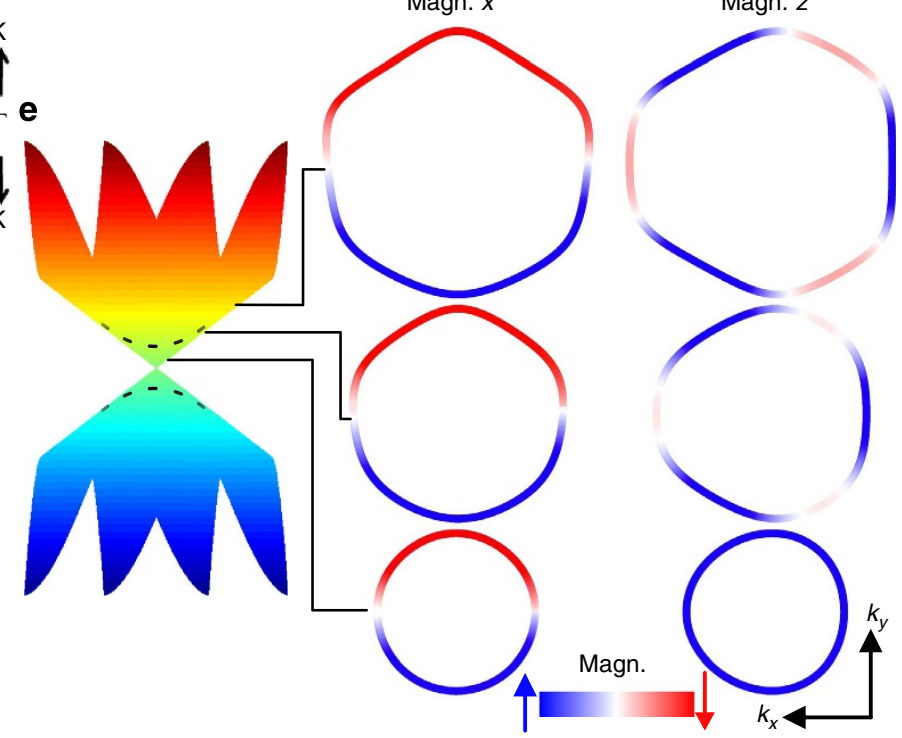

Figure 4 | Visualizing time-reversal symmetry breaking. $d / / d U$ maps with corresponding FTs obtained at energies close to the Fermi level on (a) $\mathrm{Co}$ - and (b) Mn-surface doped $\mathrm{Bi}_{2} \mathrm{Te}_{3}$. While no scattering channels are clearly visible for $\mathrm{Co}$, scattering events corresponding to back scattering emerge from the FT QPI patterns in the case of Mn. The white bars in the $\mathrm{dl} / \mathrm{dU}$ maps correspond to $20 \mathrm{~nm}$, in the FT panels to $2 \mathrm{~nm}{ }^{-1}$. The intensity of the $\mathrm{d} / / \mathrm{d} U$ signal is represented by the colour scale with red (black) and blue (white) referring to high and low signals in the $\mathrm{dl} / \mathrm{dU}$ maps (FTs), respectively. (c) ARPES constant-energy cut obtained at the Fermi level for a $\mathrm{Bi}_{2} \mathrm{Te}_{3}$ sample with a Dirac point located at $E_{\mathrm{D}}=-250 \mathrm{meV}$. From ref. 3 . Reprinted with permission from AAAS. (d) Dispersion relation obtained by analysing the backscattering channels as a function of energy. (e) Band structure and $(\mathbf{f}, \mathbf{g})$ spin textures along the magnetization direction obtained with an extended Fu model for in-plane and out-of-plane easy axes, respectively. The scale bar refers to the spin polarization along the magnetization direction with blue and red corresponding to the opposite spin.

triangular shape of Co is more pronounced (see Supplementary Fig. 2); however, here the spots indicative for TR symmetry breaking are absent. Instead, our results indicate that TR symmetry has been broken and that magnetic order is present on the surface even for a Mn concentration as low as 1\% ML.

The dispersion of $\mathbf{q}_{\mathbf{1}}$ is displayed in Fig. $4 \mathrm{~d}$. A linear fitting of the data results in a Dirac point at $E_{\mathrm{D}}=-(263 \pm 13) \mathrm{meV}$, in good agreement with the data of Fig. 3e. The origin of the magnetic order observed for $\mathrm{Mn}$ is attributed to the Dirac fermions that mediate a 2D RKKY interaction among magnetic surface impurities. The oscillation period of the RKKY interaction, which marks the transition from ferromagnetic to antiferromagnetic coupling, is determined by the Fermi wavelength $\lambda_{\mathrm{F}}$. In our experiments $\lambda_{\mathrm{F}}=7 \mathrm{~nm}$, while the average 
a

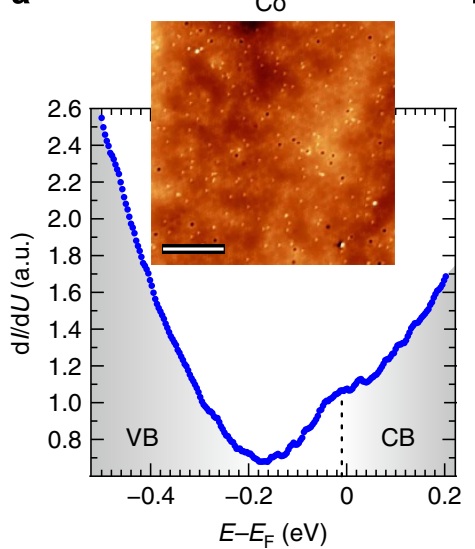

b

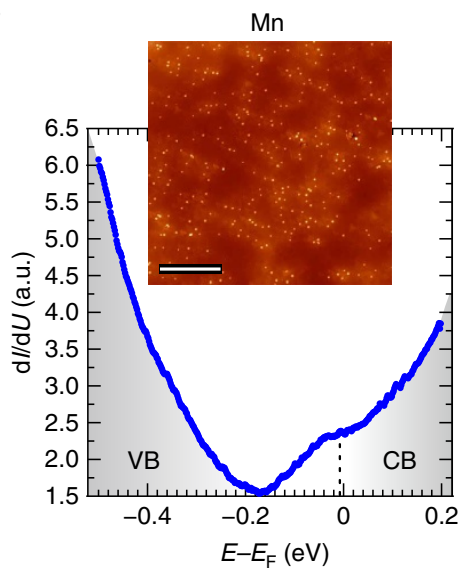

C

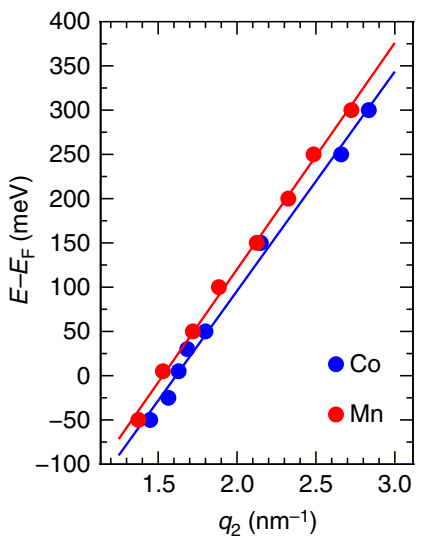

d
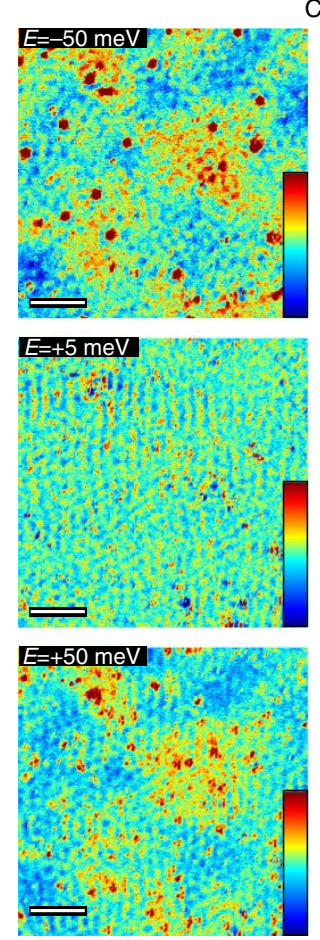

Co
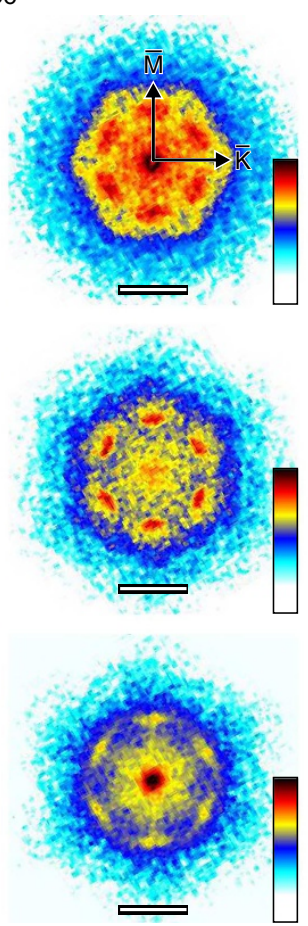

e
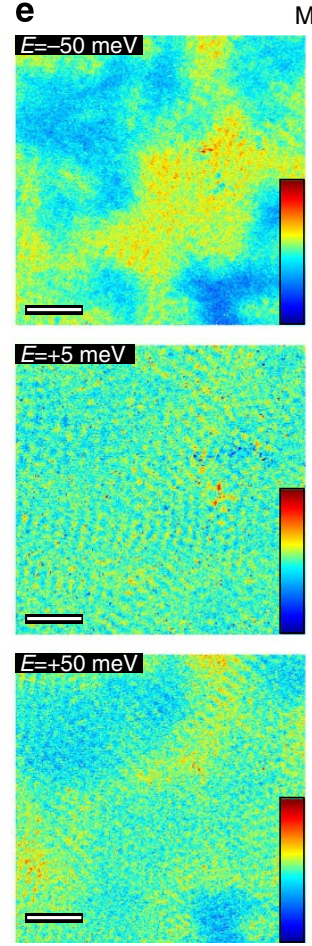

$\mathrm{Mn}$
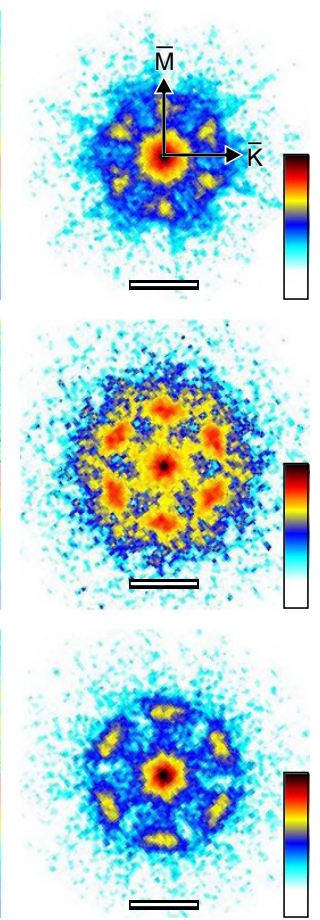

Figure 5 | Scattering channels at high doping. (a,b) STS data obtained on Co- and Mn-surface-doped $\mathrm{Bi}_{2} \mathrm{Te}_{3}$. The adatoms' concentration amounts to $\sim 2 \%$ of a ML. The insets report the corresponding constant-current images where STS have been acquired. The white scale bars correspond to $20 \mathrm{~nm}$. Note that at this coverage the Fermi level lies within the bulk conduction band. (c) A linear fit of scattering vectors provides a Dirac point positioned at -393 and $-385 \mathrm{meV}$ for $\mathrm{Co}$ and $\mathrm{Mn}$, respectively. (d,e) dl/dU maps with their FT for Co and Mn adatoms, respectively. They have been acquired on the same region displayed in the insets. No backscattering vectors appear close to the Fermi level in both cases. The white scale bars correspond to $15 \mathrm{~nm}$ in the $\mathrm{d} / / \mathrm{d} U$ maps, and $2 \mathrm{~nm}^{-1}$ in the FT panels. The intensity of the $\mathrm{dl} / \mathrm{d} U$ signal is represented by the colour scale with red (black) and blue (white) referring to high and low signals in the $\mathrm{dl} / \mathrm{d} U$ maps (FTs), respectively.

distance between nearest-neighbour adatoms amounts to $3 \mathrm{~nm}$. Therefore, although not homogeneously distributed, the magnetic moments are always ferromagnetically coupled.

On the importance of magnetic anisotropy. A detailed theoretical modelling of our experimental findings goes beyond the scope of the present work since it requires a detailed knowledge of the exchange interaction and the constant energy contours. Indeed, it has been recently shown that the effective exchange interaction among single magnetic impurities is reduced by increasing the number of electrons per atom ${ }^{37}$. Furthermore, we would like to point out that the evolution from a circle to a convex hexagon may strongly affect the behaviour of topological state in the presence of magnetic impurities since it can strongly enhance the strength of the RKKY interaction along some direction as described in ref. 38. Nevertheless, a simple picture based on an extended $\mathrm{Fu}$ model $^{39,40}$, which includes the magnetization through a term $H=J \mathbf{S} \cdot \boldsymbol{\sigma}$, where $J$ is the exchange constant that couples the electron spin $\boldsymbol{\sigma}$ to the magnetic moment $\mathbf{S}$ introduced by the magnetic adatoms (see Supplementary Note 1) can effectively explain our finding. In particular, they may be a direct consequence of the different Co and Mn singleatom anisotropy axis, which X-ray magnetic circular dichroism measurements have shown pointing in-plane and out-of plane, respectively ${ }^{25,41}$.

If the magnetization points along the out-of-plane direction, a gap opens at the Dirac point (see dashed line in Fig. $4 \mathrm{e}$ and Supplementary Fig. 6). This is not the case for an in-plane 
magnetization. Figure 4f,g reports the spin textures at different energies along the magnetization direction for in-plane and out-of-plane directions, respectively (spin texture along the magnetization direction for all directions, that is, $x, y$ and $z$, can be found in Supplementary Fig. 7). No change in the spin texture is visible for an in-plane magnetization. If magnetic moments are pointing out-of-plane, on the other hand, a spin component along the magnetization direction is introduced while preserving the in-plane spin-momentum locking. This perpendicular spin polarization is particularly strong for energies close to the Dirac point and heals when moving towards higher energies, in agreement with our experimental findings. This trend can be explained by the competition between the ferromagnetic order, which wants to keep all spins aligned along the same direction, and the out-of-plane spin texture imposed by the warping term ${ }^{40}$, which forces adjacent valleys to have opposite out-of-plane spin directions.

\section{Spectroscopic mapping of scattering channels-high coverages.} Interestingly, by continuously increasing the magnetic adatoms' concentration, backscattering disappears when the conduction band minimum is moved below the Fermi level as a consequence of the above-mentioned adatom-induced band bending. In the present case, this happens at a concentration of $\sim 2 \% \mathrm{ML}$, at which the conventional scattering scenario on TIs is recovered as shown in Fig. 5. Two different mechanisms can explain the observed behaviour. The first is that the presence of bulk states at the Fermi level marks a transition from 2D to a 3D RKKY interaction, for which the coupling strength between magnetic moments decays faster $\left(R^{-3}\right.$ with respect to the $R^{-2}$ found in $2 \mathrm{D}$ systems, $R$ being the distance between magnetic moments). The second is the larger negative shift, which increases the strength of the warping term for topological states located at the Fermi level, imposing its typical texture over that required by ferromagnetic coupling.

This mechanism may also explain why previous reports investigating the interaction of surface magnetic dopants with topological states did not detect any signature of surface magnetic order. Indeed, those experiments have been performed on $\mathrm{n}$-doped samples showing a conduction band lying well below the Fermi level, thus creating a complicating interplay between 2D and 3D states ${ }^{21-23}$.

Overall, our findings shed light on the nanoscale interaction of magnetic moments with topological states. The observations reported here show that surface-confined Dirac fermions can drive the coupling between magnetic moments at extremely dilute concentrations establishing local magnetic order. Our results pave the way to explore atomic-scale magnetism on TI surfaces and enable, through atomic manipulation or lithographic techniques, a true nanoscale engineering of topological states with arbitrary spin texture where new interesting effects may be observed.

\footnotetext{
Methods

Crystal growth. The $\mathrm{Bi}_{2} \mathrm{Te}_{3}$ single crystals have been grown by a modified Bridgman technique. $\mathrm{Bi}$ and $\mathrm{Te}$ of $99.999 \%$ purity were used for synthesis of polycrystalline $\mathrm{Bi}_{2} \mathrm{Te}_{3}$ (ref. 42). The starting composition was chosen as $60 \mathrm{~mol} \%$ of tellurium that produced the crystals with p-type conductivity and carrier concentration of $10^{18} \mathrm{~cm}^{-3}$ (ref. 42). Suspended components were sealed off in an ampoule, which was pumped down to a residual pressure of $10^{-4}$ Torr. The synthesis furnace was heated at the rate of $50 \mathrm{Kh}^{-1}$ up to $870 \mathrm{~K}$, and after $10 \mathrm{~h}$ of melt homogenization, it was switched off. Growth of single crystals was performed in carbon-coated ampoules with a $4 \mathrm{~cm}$ tip for geometrical selection of spontaneous microcrystals. The inner diameter of the ampoules was $14 \mathrm{~mm}$, while that of the tip was $4 \mathrm{~mm}$. Crystal growth procedure was carried out with a $10 \mathrm{~K} \mathrm{~cm}^{-1}$ temperature gradient at the front of crystallization. After pulling out the ampoule at the rate of $5 \mathrm{~mm}$ per day, the furnace was switched off. After growth, crystals have been cut in sizes suitable for STM experiments $(2 \mathrm{~mm}$ by $2 \mathrm{~mm}$ by $0.1 \mathrm{~mm}$ )
}

STM measurements. The experiments have been performed in an ultrahigh vacuum system equipped with a cryogenic STM. After insertion into the system, $\mathrm{Bi}_{2} \mathrm{Te}_{3}$ samples have been cleaved at room temperature at a base pressure of $3 \times 10^{-11}$ mbar and immediately inserted into the STM operated at $T=4.8 \mathrm{~K}$. All measurements have been performed using electrochemically etched tungsten tips. Spectroscopic data have been obtained using the lock-in technique and a bias voltage modulation in between 1 and $10 \mathrm{meV}$ (r.m.s.) at a frequency of $793 \mathrm{~Hz}$, with the amplitude progressively increasing with the scanning bias. $\mathrm{d} I / \mathrm{d} U$ maps have been acquired simultaneously to topographic images in constant current mode. Before Fourier transformation, the average value has been subtracted to each $\mathrm{d} I / \mathrm{d} U$ map. To increase the signal-to-noise ratio, FT $\mathrm{d} I / \mathrm{d} U$ maps have been threefold symmetrized according to the rotational symmetry of the underlying surface. Co and Mn (Alfa Aesar) were deposited using an e-beam evaporator with the sample kept at $T=5.3 \mathrm{~K}$ at an evaporation rate of $1 \% \mathrm{ML}$ per min. Co and $\mathrm{Mn}$ purity was verified by looking at the Kondo state at the $\operatorname{Ag}(111)$ surface $^{43}$ and at the antiferromagnetic ground state of $\mathrm{Mn}$ islands on $\mathrm{W}(110)^{44}$, respectively.

\section{References}

1. Romming, N. et al. Writing and deleting single magnetic skyrmions. Science 341, 636-639 (2013).

2. Hsieh, H. et al. A topological Dirac insulator in a quantum spin Hall phase. Nature 452, 970-974 (2008).

3. Chen, Y. L. et al. Experimental realization of a three-dimensional topological insulator, $\mathrm{Bi}_{2} \mathrm{Te}_{3}$. Science 325, 178-181 (2009).

4. Fu, L. \& Kane, C. L. Superconducting proximity effect and majorana fermions at the surface of a topological insulator. Phys. Rev. Lett. 100, 096407 (2008).

5. Qi, X.-L., Li, R., Zang, J. \& Zhang, S.-C. Inducing a magnetic monopole with topological surface states. Science 323, 1184-1187 (2009).

6. Garate, I. \& Franz, M. Inverse spin-galvanic effect in the interface between a topological insulator and a ferromagnet. Phys. Rev. Lett. 104, 146802 (2010).

7. Yokoyama, T., Tanaka, Y. \& Nagaosa, N. Giant spin rotation in the junction between a normal metal and a quantum spin hall system. Phys. Rev. Lett. 102, 166801 (2009).

8. Tanaka, Y., Yokoyama, T. \& Nagaosa, N. Manipulation of the Majorana Fermion, Andreev Reflection, and Josephson current on topological insulators Phys. Rev. Lett. 103, 107002 (2009).

9. Akhmerov, A. R., Nilsson, J. \& Beenakker, C. W. J. Electrically detected interferometry of Majorana fermions in a topological insulator. Phys. Rev. Lett 102, 216404 (2009).

10. Novoselov, K. S. et al. Electric field effect in atomically thin carbon films. Science 306, 666-669 (2004).

11. Bianchi, M. et al. Coexistence of the topological state and a two-dimensional electron gas on the surface of $\mathrm{Bi}_{2} \mathrm{Se}_{3}$. Nat. Commun. 1, 128 (2010).

12. Benia, H. M., Lin, C., Kern, K. \& Ast, C. R. et al. Reactive chemical doping of the Bi2Se3 topological insulator. Phys. Rev. Lett. 107, 177602 (2011).

13. Hsieh, D. et al. Observation of unconventional quantum spin textures in topological insulators. Science 323, 919-922 (2009).

14. Roushan, P. et al. Topological surface states protected from backscattering by chiral spin texture. Nature 460, 1106-1109 (2009).

15. Zhang, T. et al. Experimental demonstration of topological surface states protected by time-reversal symmetry. Phys. Rev. Lett. 103, 266803 (2009)

16. Chen, Y. L. et al. Massive Dirac Fermion on the surface of a magnetically doped topological insulator. Science 329, 659-662 (2010).

17. Hor, Y. S. et al. Development of ferromagnetism in the doped topological insulator $\mathrm{Bi}_{2-\mathrm{x}} \mathrm{Mn}_{\mathrm{x}} \mathrm{Te}_{3}$. Phys. Rev. B 81, 195203 (2010).

18. Xu, S.-Y. et al. Hedgehog spin texture and Berry's phase tuning in a magnetic topological insulator. Nat. Phys. 8, 616-622 (2012).

19. Okada, Y. et al. Direct observation of broken time-reversal symmetry on the surface of a magnetically doped topological insulator. Phys. Rev. Lett. 106, 206805 (2011)

20. Checkelsky, J. G. et al. Dirac-fermion-mediated ferromagnetism in a topological insulator. Nat. Phys. 8, 729-733 (2012)

21. Wray, L. A. et al. A topological insulator surface under strong Coulomb, magnetic and disorder perturbations. Nat. Phys. 7, 32-37 (2011).

22. Valla, T., Pan, Z.-H., Gardner, D., Lee, Y. S. \& Chu, S. Photoemission spectroscopy of magnetic and nonmagnetic impurities on the surface of the $\mathrm{Bi}_{2} \mathrm{Se}_{3}$ topological insulator. Phys. Rev. Lett. 108, 117601 (2012).

23. Scholz, M. R. et al. Tolerance of topological surface states towards magnetic moments: $\mathrm{Fe}$ on $\mathrm{Bi}_{2} \mathrm{Se}_{3}$. Phys. Rev. Lett. 108, 256810 (2012).

24. Honolka, J. et al. In-plane magnetic anisotropy of $\mathrm{Fe}$ atoms on $\mathrm{Bi}_{2} \mathrm{Se}_{3}(111)$. Phys. Rev. Lett. 108, 256811 (2012).

25. Shelford, L. R. et al. Electronic structure of Fe and Co magnetic adatoms on $\mathrm{Bi}_{2} \mathrm{Te}_{3}$ surfaces. Phys. Rev. B 86, 081304(R) (2012).

26. Liu, Q., Liu, C.-X., Xu, C., Qi, X.-L. \& Zhang, S. C. Magnetic impurities on the surface of a topological insulator. Phys. Rev. Lett. 102, 156603 (2009).

27. Abanin, D. A. \& Pesin, D. A. Ordering of magnetic impurities and tunable electronic properties of topological insulators. Phys. Rev. Lett. 106, 136802 (2011). 
28. Crommie, M. F., Lutz, C. P. \& Eigler, D. M. Imaging standing waves in a twodimensional electron gas. Nature 363, 524-527 (1993).

29. Hoffman, J. E. et al. Imaging Quasiparticle Interference in $\mathrm{Bi}_{2} \mathrm{Sr}_{2} \mathrm{CaCu}_{2} \mathrm{O}_{8+\delta}$. Science 297, 1148-1151 (2002).

30. Schmidt, A. R. et al. Imaging the Fano lattice to 'hidden order' transition in $\mathrm{URu}_{2} \mathrm{Si}_{2}$. Nature 465, 570-576 (2010).

31. Aynajian, P. et al. Visualizing heavy fermions emerging in a quantum critical Kondo lattice. Nature 486, 201-206 (2012).

32. Pascual, J. I. et al. Role of spin in quasiparticle interference. Phys. Rev. Lett. 93, 196802 (2004).

33. El-Kareh, L., Sessi, P., Bathon, T. \& Bode, M. Quantum interference mapping of Rashba-split bloch states in Bi/Ag(111). Phys. Rev. Lett. 110, 176803 (2013).

34. Simon, L., Bena, C., Vonau, F., Cranney, M. \& Aubel, D. Fourier-transform scanning tunneling spectroscopy: the possibility to obtain constant-energy maps and band dispersion using a local measurement. J. Phys. D Appl. Phys. 44, 464010 (2011).

35. Alpichshev, Z. et al. STM imaging of electronic waves on the surface of Bi2Te3: topologically protected surface states and hexagonal warping effects. Phys. Rev. Lett. 104, 016401 (2009).

36. Beidenkopf, H. et al. Spatial fluctuations of helical Dirac fermions on the surface of topological insulators. Nat. Phys. 7, 939-943 (2011).

37. Vergniory, M. G. et al. Exchange interaction and its tuning in magnetic binary chalcogenides. Phys. Rev. B 89, 165202 (2014).

38. Roth, L. M., Zeiger, H. J. \& Kaplan, T. A. Generalization of the RudermanKittel-Kasuya-Yosida interaction for nonspherical Fermi surfaces. Phys. Rev. 149, 519 (1966)

39. $\mathrm{Fu}, \mathrm{L}$. Hexagonal warping effects in the surface states of the topological insulator $\mathrm{Bi}_{2} \mathrm{Te}_{3}$. Phys. Rev. Lett. 103, 266801 (2009).

40. Henk, J. et al. Topological character and magnetism of the dirac state in Mn-doped $\mathrm{Bi}_{2} \mathrm{Te}_{3}$. Phys. Rev. Lett. 109, 076801 (2009).

41. Eelbo, T. et al. Magnetic Dopants and Adatoms in and on $\mathrm{Bi}_{2} \mathrm{Se}_{3}$ and $\mathrm{Bi}_{2} \mathrm{Te}_{3}$ Single Crystals Studied with XMCD. ESRF Report http://ftp.esrf.eu/pub/ UserReports/47676_A.pdf (2012).
42. Kokh, K. A., Makarenko, S. V., Golyashov, V. A., Shegai, O. A. \& Tereshenko, O. E. Melt growth of bulk Bi2Te3 crystals with a natural p-n junction. Cryst. Eng. Commun. 16, 581 (2014).

43. Schneider, M. A. et al. Kondo state of Co impurities at noble metal surfaces. Appl. Phys. A 80, 937-941 (2005).

44. Sessi, P., Guisinger, N. P., Guest, J. R. \& Bode, M. Temperature and size dependence of antiferromagnetism in Mn nanostructures. Phys. Rev. Lett. 103, 167201 (2009).

\section{Acknowledgements}

P.S. gratefully acknowledges discussions with P. Michetti.This work was supported by the Deutsche Forschungsgemeinschaft within SPP 1666 (Grant No. BO1468/21-1). K.A.K. and O.E.T. acknowledge the financial support by the RFBR (Grant nos. 13-02-92105 and 14-08-31110)

\section{Author contributions}

P.S. conceived the experiment. P.S., F.R. and T.B. performed and analysed the measurements. P.S. modelled the data. K.A.K. and O.E.T. synthesized the crystal. P.S. and M.B. wrote the manuscript. M.B. supervised the project. All authors discussed and commented on the manuscript.

\section{Additional Information}

Supplementary Information accompanies this paper at http://www.nature.com/ naturecommunications

Competing financial interests: The authors declare no competing financial interest.

Reprints and permission information is available online at http://npg.nature.com/ reprintsandpermissions/

How to cite this article: Sessi, P. et al. Signatures of Dirac fermion-mediated magnetic order. Nat. Commun. 5:5349 doi: 10.1038/ncomms6349 (2014). 